\title{
A comprehensive map of the variability in the Lipoprotein(a) KIV-2 repeat region and follow-up of the KIV-2 Arg20Ter mutation in 11,000 individuals.
}

Stefan Coassin ${ }^{1}$, Sebastian Schönherr 1, Hansi Weissensteiner 1, Gertraud Erhart 1, Silvia Di Maio 1, Claudia Lamina 1, Lukas Forer 1 , Annette Peters ${ }^{2}$, Barbara Thorand ${ }^{2}$, Kai-Uwe Eckardt ${ }^{3}$, Anna Köttgen ${ }^{4}$, Gerd Utermann ${ }^{5}$, Günther Specht ${ }^{6}$, Florian Kronenberg ${ }^{1}$

1 Division of Genetic Epidemiology, Medical University of Innsbruck, Innsbruck, Austria. ${ }^{2}$ Institute of Epidemiology, Helmholtz Zentrum München, Neuherberg, Germany; ${ }^{3}$ Department of Nephrology and Medical Intensive Care, Charité - Universitätsmedizin Berlin, Berlin, Germany; ${ }^{4}$ Department of Biometry, Epidemiology and Medical Bioinformatics, Institute of Genetic Epidemiology, University of Freiburg, Freiburg, Germany. ${ }^{5}$ Division of Human Genetics, Medical University of Innsbruck, Innsbruck, Austria; ${ }^{6}$ DBIS, Institute of Computer Science, University of Innsbruck, Innsbruck, Austria

\section{Background}

- The LPA gene locus controls $>90 \%$ of Lipoprotein(a) [Lp(a)] variance ${ }^{1}$.

The KIV-2 repeat $(5.6 \mathrm{~kb}$ ) explains $40-70 \%$ of $L p(a)$ variance.

However, individual $L p(a)$ levels still vary by up to 1000 -fold and a sizeable proportion of $L p(a)$ variance is still unexplained. Moreover, null alleles (i.e. isoforms not expressed in plasma) are common .

The KIV-2 repeat region has never been screened for mutations on a large scale ${ }^{3,5,6}$, although it contains up to $70 \%$ of the protein coding sequence. It thus represents a natural candidate region for novel variants affecting $L p(a)$ concentrations.

\section{Project summary}

We developed a dedicated NGS sequencing pipeline for the KIV-2 region, cataloged the variability in 123 Caucasian samples and 2,504 samples from 1000 Genomes and followed up the loss-of-function variant Arg20Ter in 11,152 individuals.

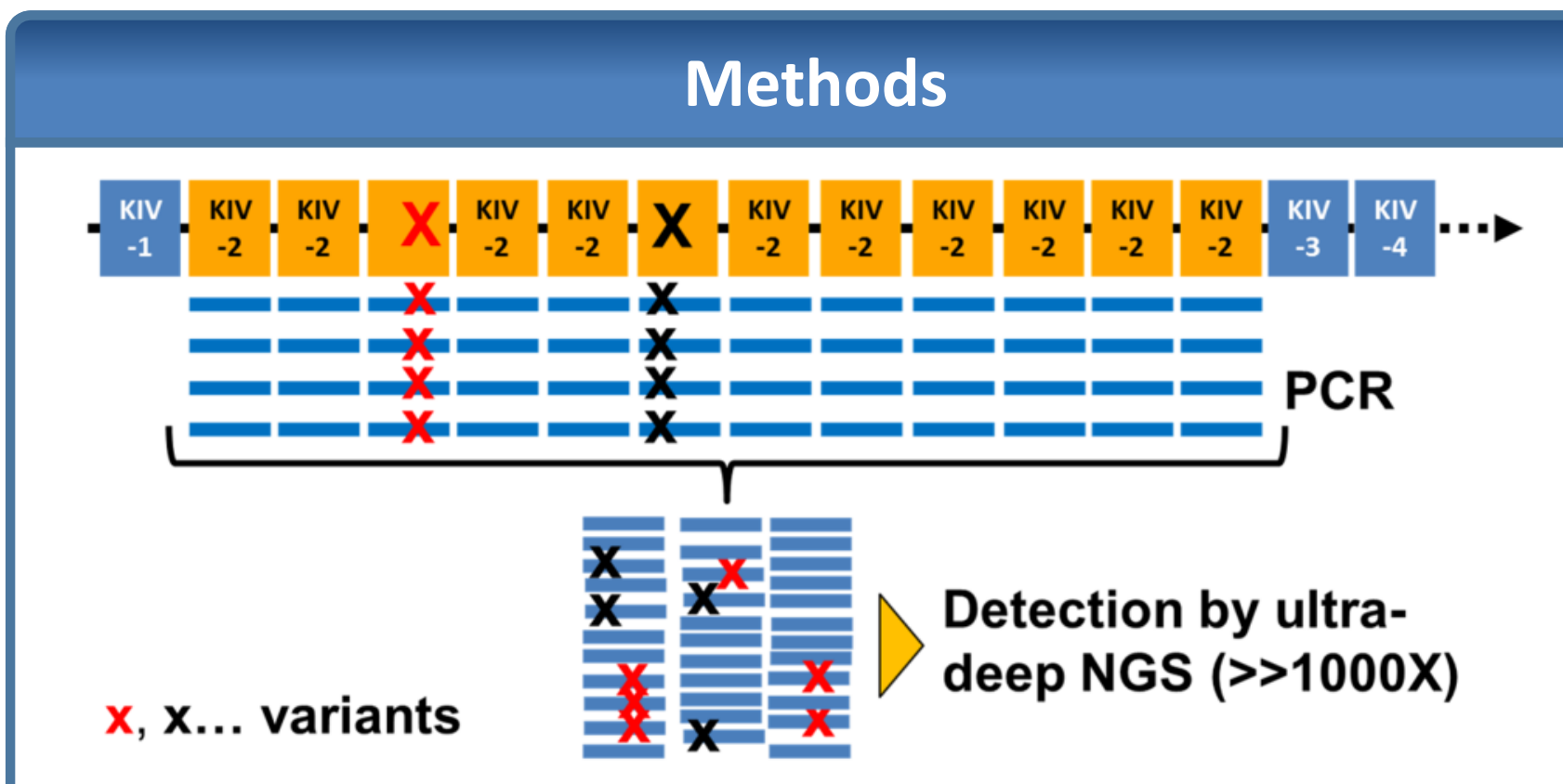

Ultra-deep "batch NGS sequencing": All KIV-2 are amplified as a mixture using primer binding to all repeats ("batch sequencing") $)^{2-4}$ and collectively ultra-deep sequenced (Illumina MiSeq v2, TrueSeq Nano, average depth: 24,418X). 123 Caucasian samples from Austria and Germany were sequenced.

Data analysis: We created a dedicated low level variant caller for the KIV-2 region ${ }^{5}$, which takes into account several sequence peculiarities of this region. All reads are mapped to one KIV-2 repeat (hg19 chr6:161,033,785-161,038,888). Variants $\geq 1 \%$ mutational level were called (see Ref). Additionally, the public sequence data from the 1000 Genomes project ( 26 populations worldwide) has been reanalyzed ( $n=2,504$ exomes, $n=150$ for intronic regions)

Follow up of Arg20Ter: Carrier status for the Arg20Ter ${ }^{6}$ loss-of-function was been assessed in 4 populations: GCKD (German Chronic Kidney Disease Study, $n=4771$ ), KORA F3 ( $n=3099$ ) and F4 ( $n=3040$. both: general populations from Southern Germany) using a high throughput, allele-specific TaqMan qPCR protocol. The assay showed sensitivity down to $0.5 \%$ mutation level in plasmid mixes.

Association study: Wilcoxon test, linear regression (unadjusted and isoform-adjusted) and fixed-effect meta-analysis. Lp(a) levels were determined by ELISA, apo(a) isoforms by Western Blotting.

Githe variant calling pipeline for the LPA KIV-2 region is freely available at https://github.com/genepi/lpa-pipeline

The KIV-2 repeat contains hundreds of variants

Table 1. Number of variants found

\begin{tabular}{lccc}
\hline & $\begin{array}{c}\mathbf{1 0 0 0} \text { Genomes } \mathbf{n = 2 , 5 0 4} \\
\text { (introns: } \mathbf{n = 1 5 0 )}\end{array}$ & $\begin{array}{c}\text { Discovery set } \\
\mathbf{n = 1 2 3}\end{array}$ & Overlap \\
\hline Nonsense & 9 & 2 & 1 \\
Missense \& nonsense & 115 & 12 & 11 \\
Synonymous & 56 & 11 & 8 \\
Splice site & 3 & 0 & 0 \\
Non-coding & 937 & 488 & 278 \\
\hline
\end{tabular}

Ref.: Coassin S., Schoenherr S. et al, A comprehensive map of single-base polymorphisms in the hypervariable LPA kringle IV type 2 copy number variation region. J Lipid Res. 60: 186-199, 2019

Funding: D•A•CCH Advancement Award Lipidology 2015 of the D•A•CCH-Society Prevention of Cardiovascular Diseases, Austrian Science Fund (FWF) P 31458 ;
F.K.: Austrian Genome Project 'GOLD' to F.K.; C.L.: FWF P 266600-B13; GCKD study: BMBF grants 01ER 0804, 01ER 0818, 01ER 0819, $01 E R$ 0820 and $01 E R 0821$ and the KFH Foundation for Preventive Medicine; KORA studies: initiated and financed by the Helmholtz Zentrum München - German
Research Center for Environmental Health, the State of Bavaria and LMUinnovativ (Munich Center of Health Sciences (MCHealth), LMU Munich)

\section{Some positions are variable in every individual}

Figure 1: Number of discovery samples $(n=123)$ with a variant at a given position. The KIV-2 Dralll polymorphism (Mancini et al, 1995) was identified as a 2106 G>A. Black: non-coding; blue: splice region ( \pm 25 bp), orange: synonymous, red: missense and nonsense.

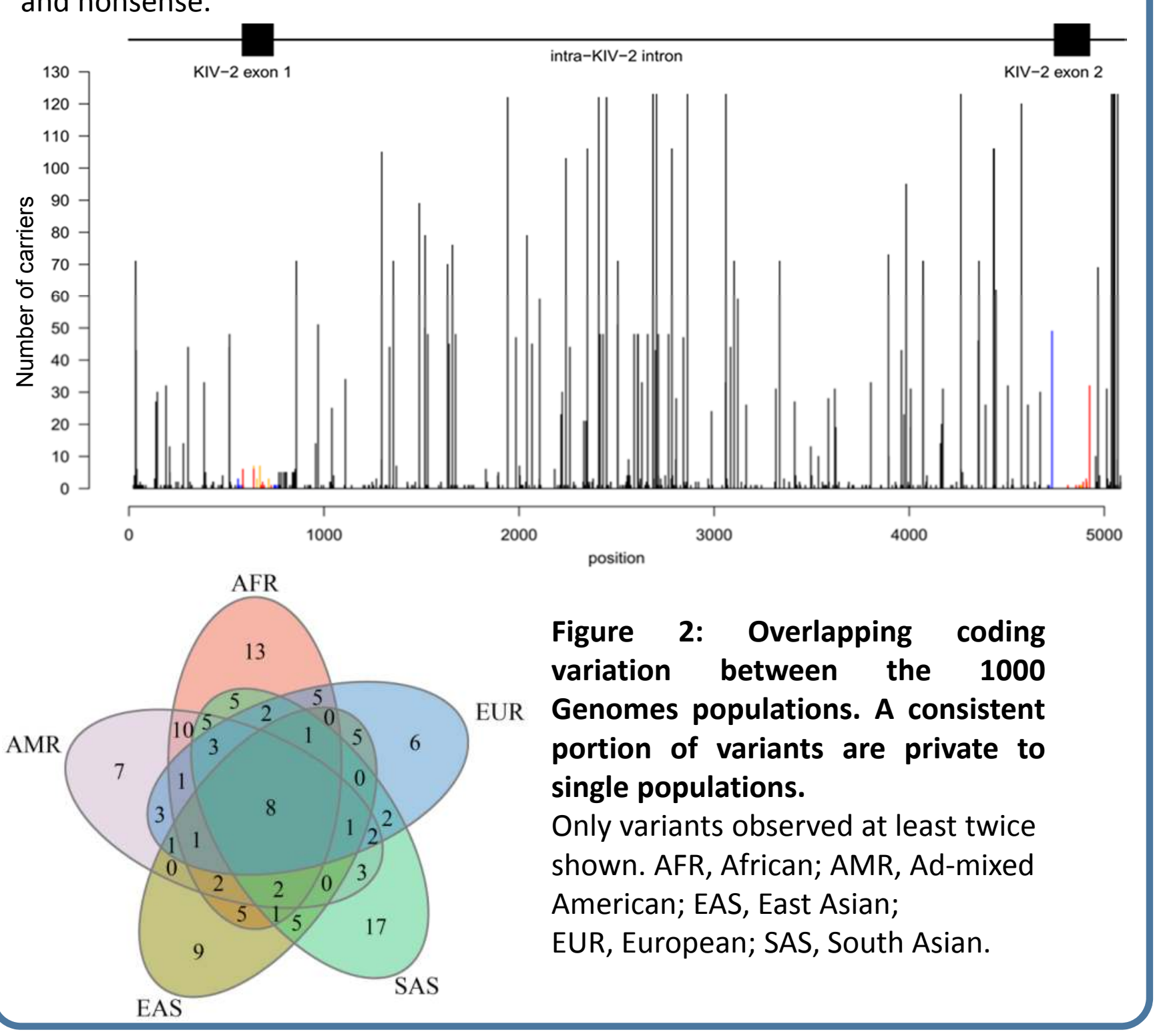

KIV-2 Arg20Ter decreases Lp(a) by $11.7 \mathrm{mg} / \mathrm{dL}$

Table 2: Association results for Arg20Ter ${ }^{6}$ and plasma Lp(a).

* Original scale $(\mathrm{mg} / \mathrm{dL}), \dagger$ Inverse normal scale

$$
\begin{array}{l|l|l|l|l}
\text { Population } & \text { carriers } & \text { total } n & \beta[\mathrm{Cl}]^{*} & \mathrm{SE} \\
\end{array}
$$

Age and sex adjusted model for $L p(a)$ plasma concentrations

\begin{tabular}{cccrccc} 
GCKD & 74 & 4,771 & $-13.0[-20.0,-6.0]$ & 3.58 & $4.3 \mathrm{e}-12$ & 0.0114 \\
KORA F3 & 56 & 3,099 & $-12.6[-19.5,-5.7]$ & 3.51 & $6.5 \mathrm{e}-11$ & 0.0132 \\
KORA F4 & 63 & 3,040 & $-9.9[-16.0,-3.8]$ & 3.12 & $4.0 \mathrm{e}-12$ & 0.0153 \\
\hline Meta analysis & $\mathbf{2 1 1}$ & $\mathbf{1 0 , 9 1 0}$ & $\mathbf{- 1 1 . 7}[-\mathbf{1 5 . 5}, \mathbf{- 7 . 8}]$ & $\mathbf{1 . 9 6}$ & $\mathbf{1 . 1 e - 3 2}$ & -
\end{tabular}

- No SNP in perfect LD found among our recent GWAS hits, but all Arg20Ter carriers carry also the frequent KIV-8 splice site mutation rs41272114 ${ }^{7,8}\left(D^{\prime}=0.957, R^{2}=0.275\right)$. Not applicable vice versa.

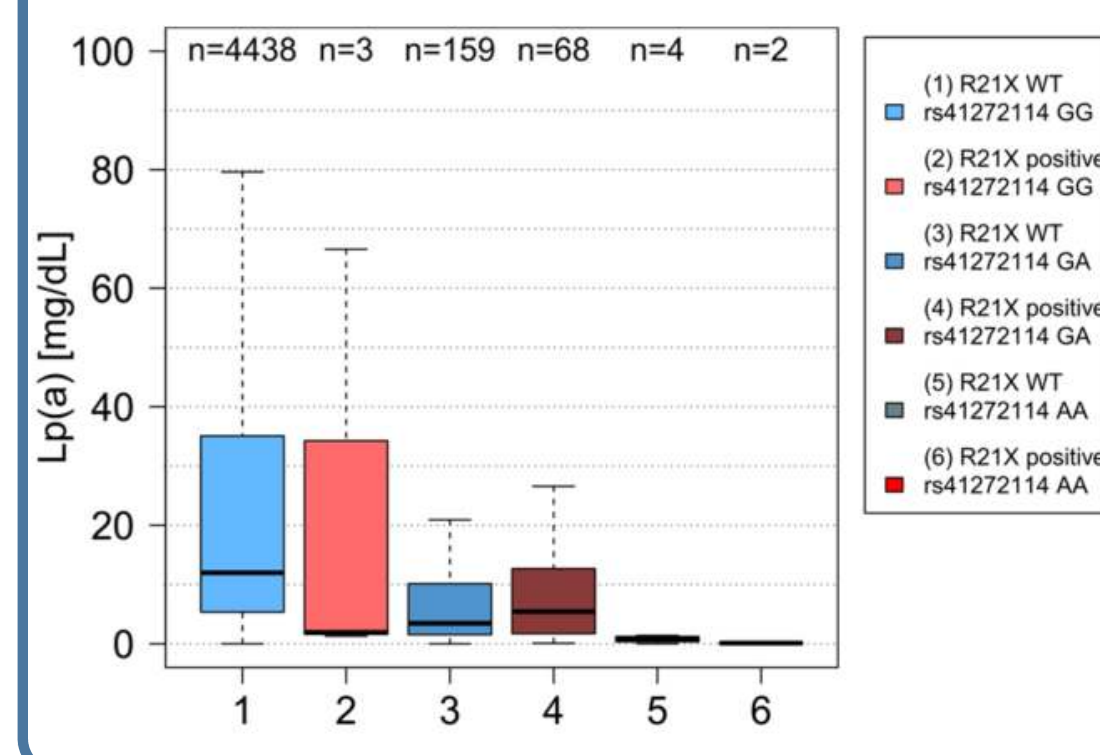

Figure 3: Box plot of the Lp(a) levels observed in the various combinations of R21X carrier status (WT or positive) and rs41272114 genotypes.

The $p$ value between group 3 and 4 is $p=0.47$ (Wilcoxon test). The two variants do not have an additive effect, their effects cannot be separated.

\section{Main findings}

Ultra-deep sequencing allows detection of variants in the KIV-2.

The KIV-2 region is highly variable containing hundreds of variants.

Arg20Ter is moderately frequent in Caucasians and associated with decreased $L p(a)(-11.7 \mathrm{mg} / \mathrm{dL})$, explaining 1.1-1.5\% of $L p(a)$ variance.

Virtually all Arg20Ter carriers carry also rs41272114. The effects cannot be separated. Arg20Ter alleles represent "double null alleles". 